\section{Idiopathic or connective tissue disease-associated interstitial lung disease: a case of HRCT mimicry}

\author{
Kevin R Flaherty, ${ }^{1}$ Dinesh Khanna ${ }^{2}$
}

Interstitial lung disease (ILD) induces overwhelming morbidity and kills more patients with connective tissue disease (CTD) than any other CTD-related manifestation. $^{1-5}$ Because of this, there is a keen and growing interest in the rheumatology and pulmonary communities to identify prognostic variables for CTDassociated ILD (CTD-ILD). Treatment options for patients with ILD, idiopathic as well as those associated with CTD-ILD are limited. Determining prognosis is important, especially when considering therapeutic options such as lung transplantation as well as monitoring response to therapy.

High-resolution CT (HRCT) plays a central role in the diagnostic evaluation of patients with ILDs. ${ }^{6}$ Furthermore, features such as honeycombing and severity of fibrosis can both serve as diagnostic surrogates for surgical lung biopsy as well as predict the risk of subsequent mortality for patients with idiopathic interstitial pneumonias (IIP). ${ }^{7-9}$ Less is published regarding the prognostic characteristics of features seen on HRCT in patients with CTD-ILD; diseases that often have a better prognosis compared with patients with IIP ${ }^{10}{ }^{11}$ and where surgical lung biopsy is less often performed.

Walsh $e t a l^{12}$ evaluated the ability of radiographic and pulmonary function characteristics to predict subsequent prognosis in patients with CTD-ILD. They included a relatively large and diverse study population consisting of 168 patients with a variety of CTDs such as rheumatoid arthritis $(n=39)$, systemic sclerosis (SSc) $(n=32)$, mixed CTD $(n=33)$, polymositis $(n=33)$, systemic lupus erythematosus $(n=17)$ and Sjogren's disease $(n=14)$. Fifty-one patients had surgical lung biopsy available for evaluation.

\footnotetext{
${ }^{1}$ Division of Pulmonary/Critical Care Medicine, Department of Internal Medicine, University of Michigan, Ann Arbor, Michigan, USA; ${ }^{2}$ Division of Rheumatology, Department of Internal Medicine, University of Michigan, Ann Arbor, Michigan, USA

Correspondence to Dr Kevin R Flaherty, Division of Pulmonary/Critical Care Medicine, Department of Internal Medicine, University of Michigan, 1500 E. Medical Center Drive, 3916 Taubman Center, Ann Arbor, MI 48109, USA; flaherty@umich.edu
}

The initial radiographic assessment was rigorous and complex. HRCTs were scored by two experienced thoracic radiologists for the presence of ground glass opacification, reticulation, honeycombing, consolidation and extent of traction bronchiectasis. Each feature was scored at six specified levels. At each of these levels, the extent of disease pattern was estimated to the nearest 5\% and summed to $100 \%$. Traction bronchiectasis was scored semiquantitatively as 0 -none, 1 -mild (borderline traction bronchiectasis), 2moderate (definite traction bronchiectasis but not severe) and 3-severe. If the estimated total disease extent differed by more than $5 \%$ or traction bronchiectasis score differed by more than one point, the radiologist reviewed the case to reach a consensus. The radiologists also calculated scores for overall total disease extent, disease extent for each pattern and traction bronchiectasis.

Similar to what has been reported with IIP, radiographic features in CTD-ILD also predicted survival with extent of honeycombing, traction bronchiectasis and diffusion capacity for carbon monoxide all remaining significant in multivariable analysis. As above, the HRCT scores were derived by expert radiologists who met by consensus to resolve discrepancies. The requirement for the detailed scoring performed in this study would certainly limit the applicability of these data in general practice. Fortunately, the investigators also evaluated a simple binary score for the presence/absence of honeycombing and traction bronchiectasis. In multivariable analysis, these binary scores were also significant with the presence of traction bronchiectasis (HR 4.0, 95\% CI 1.19 to $13.38, \mathrm{p}=0.001$ ), honeycombing ( $\mathrm{HR}$ $2.87,95 \%$ CI 1.53 to $5.43, \mathrm{p}=0.022$ ) and diffusion capacity all remaining significant predictors of increased risk of subsequent mortality. The prognostic strength of binary scores was preserved on subgroup analysis where each type of CTD subgroup was removed from the remaining cohort-this lends strength that no single CTD was driving the results and these are likely generalisable across the CTDs present in the study.
Investigators also evaluated the concordance of HRCT diagnostic category (usual interstitial pneumonia, UIP, indeterminate and fibrotic non-specific interstitial pneumonia, NSIP) with histopathologic pattern in a subgroup of 51 patients. Similar to published results in IIP, $^{7}{ }^{13}$ the concordance of a HRCT pattern of UIP with histologic pattern of UIP was excellent, while HRCT patterns of indeterminate or NSIP showed both NSIP and UIP at biopsy. These results extend previously published findings ${ }^{14}$ and highlight that in patients with both IIP and CTD-ILD a HRCT pattern of UIP is highly predictive of a pattern of UIP at surgical lung biopsy, while the predictive ability of HRCT to accurately define other histopathologic patterns, such as NSIP, is lacking.

The impact of radiographic disease category alone and combined with surgical lung biopsy patterns was also evaluated. Interobserver agreement for determining HRCT pattern was good with a weighted $\kappa$ of 0.74 . Similar to IIPs $^{7} 13$ the radiographic pattern of UIP conferred an increased risk of mortality compared with patterns of indeterminate or NSIP. This finding also held when looked at in a subgroup that had surgical lung biopsy information with patients who showed concordant UIP (biopsy and HRCT UIP) having worse prognosis than discordant UIP (incongruent biopsy and HRCT patterns) which were in turn worse than concordant NSIP.

In another analysis of 215 SSc patients from the same group followed for 10 years, ${ }^{15}$ baseline pulmonary function tests and total lung involvement on HRCTs were predictive of mortality risk. An increased extent of disease (as defined by the presence of ground glass opacity, fibrosis or bronchiectasis) on HRCT $>20 \%$ associated with an increase in mortality (HR 2.48, $\mathrm{p}<0.0005)$. Patients with a decreased baseline FVC $<70 \%$ also had increased mortality risk (HR 2.11, $\mathrm{p}=0.001)$. When the two modalities were combined in the risk assessment, patients with HRCT extent of $\geq 10 \%$ in addition to a FVC $<70 \%$ had the highest mortality risk (HR 3.46, $\mathrm{p}<0.0005)$. It is surprising that the authors did not look at staging system combining HRCT and pulmonary physiologic parameters in the current work.

The authors do not provide the disease duration of the CTD. At least in SSc, ILD occurs early in the disease (usually within the first 5-6 years). Analysis from the Scleroderma Lung Study-1, a multicentre, double-blind, randomised controlled study to evaluate the effectiveness and safety of oral cyclophosphamide 
administered for 1 year in 158 patients with early symptomatic SSc-ILD who had evidence of active alveolitis either by analysis of bronchoalveolar lavage ( $\geq 3 \%$ neutrophils and/or $\geq 2 \%$ eosinophils) and/or thoracic HRCT (any ground glass opacification) found that the most common HRCT findings in SSc-related ILD were reticulation (93\%), ground glass opacities (90\%) and honeycombing (37\%). ${ }^{16}$ The extent of fibrosis seen on baseline HRCT scan was predictive of the progressive rate of decline in FVC in subjects not receiving active treatment, ${ }^{17}$ as well as the response to cyclophosphamide therapy. ${ }^{18}$ Patients with the most extensive fibrosis seen on baseline HRCT scans responded the greatest to cyclophosphamide treatment. ${ }^{19}$ Both in the Scleroderma Lung Study and in the current study, ground glass opacities were not predictive of decline in $\mathrm{FVC} \%$ or $\mathrm{D}_{\mathrm{L}} \mathrm{CO} \%$ predicted $^{17}$ and survival, respectively. The current study does not provide data on immunosuppressive treatment provided to the patients or impact of treatment of stabilisation of ILD or survival.

In summary, the study by Walsh et al demonstrates that much of what we know about the prognostic ability of HRCT for patients with IIP also applies to patients with CTD-ILD. A radiographic pattern of UIP confers a high likelihood of finding a pattern of UIP at biopsy and portents a worse prognosis compared with HRCT patterns that are indeterminate or show NSIP. It also appears that the simple presence of traction bronchiectasis, a marker of contraction and fibrosis, may be the single most important HRCT feature in determining prognosis.

Competing interests DK was supported by $\mathrm{NIH} /$ NIAMS K24AR063120-02.
KRF was supported by NIH/NHLBI T32HL00749, K24PA10-061, R01HL091743, RC2 HL101740-01, P50 HL107177 and U01 HL105371.

Provenance and peer review Not commissioned; internally peer reviewed.

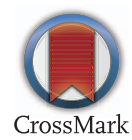

To cite Flaherty KR, Khanna D. Thorax 2014;69:205206.

Received 19 November 2013

Accepted 22 November 2013

Published Online First 6 January 2014

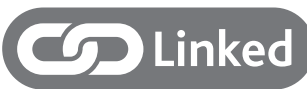

http://dx.doi.org/10.1136/thoraxjnl-2013-203843

Thorax 2014;69:205-206

doi:10.1136/thoraxjnl-2013-204864

\section{REFERENCES}

1 Connors GR, Christopher-Stine L, Oddis CV, et al. Interstitial lung disease associated with the idiopathic inflammatory myopathies: what progress has been made in the past 35 years? Chest 2010;138:1464-74.

2 Danko K, Ponyi A, Constantin T, et al. Long-term survival of patients with idiopathic inflammatory myopathies according to clinical features: a longitudinal study of 162 cases. Medicine 2004:83:35-42.

3 Khanna D, Denton CP. Evidence-based management of rapidly progressing systemic sclerosis. Best Pract Res Clin Rheumatol 2010;24:387-400.

4 Steen VD, Conte C, Owens GR, et al. Severe restrictive lung disease in systemic sclerosis. Arthritis Rheum 1994;37:1283-9.

5 Steen VD, Medsger TA. Changes in causes of death in systemic sclerosis, 1972-2002. Ann Rheum Dis 2007:66:940-4

6 Raghu G, Collard HR, Egan JJ, et al. An official ATS/ ERS/JRS/ALAT statement: idiopathic pulmonary fibrosis: evidence-based guidelines for diagnosis and management. Am J Respir Crit Care Med 2011;183:788-824.
7 Flaherty KR, Thwaite EL, Kazerooni EA, et al. Radiological versus histological diagnosis in UIP and NSIP: survival implications. Thorax 2003;58: $143-8$.

8 Lee $\mathrm{HY}$, Lee $\mathrm{KS}$, Jeong YJ, et al. High-resolution CT findings in fibrotic idiopathic interstitial pneumonias with little honeycombing: serial changes and prognostic implications. AJR Am J Roentgenol 2012;199:982-9..

9 Lynch DA, Godwin JD, Safrin S, et al. High-resolution computed tomography in idiopathic pulmonary fibrosis: diagnosis and prognosis. Am J Respir Crit Care Med 2005;172:488-93.

10 Flaherty KR, Colby TV, Travis WD, et al. Fibroblastic foci in usual interstitial pneumonia: idiopathic versus collagen vascular disease. Am J Respir Crit Care Med 2003; 167:1410-15.

11 Park JH, Kim DS, Park IN, et al. Prognosis of fibrotic interstitial pneumonia: idiopathic versus collagen vascular disease-related subtypes. Am J Respir Crit Care Med 2007:175:705-11.

12 Walsh S, Sverzellati N, Devaraaj A, et al. Connective tissue disease related fibrotic lung disease: HRCT paterns and pulmonary function indices as prognostic determinants. Thorax 2014:69:216-22.

13 Monaghan H, Wells AU, Colby TV, et al. Prognostic implications of histologic patterns in multiple surgical lung biopsies from patients with idiopathic interstitial pneumonias. Chest 2004;125:522-6.

14 Vij R, Noth I, Strek ME. Autoimmune-featured interstitial lung disease: a distinct entity. Chest 2011;140:1292-9.

15 Goh NS, Desai SR, Veeraraghavan S, et al. Interstitial lung disease in systemic sclerosis: a simple staging system. Am J Respir Crit Care Med 2008:177:1248-54.

16 Goldin JG, Lynch DA, Strollo DC, et al. High-resolution CT scan findings in patients with symptomatic scleroderma-related interstitial lung disease. Chest 2008;134:358-67.

17 Khanna D, Tseng CH, Farmani N, et al. Clinical course of lung physiology in patients with scleroderma and interstitial lung disease: analysis of the Scleroderma Lung Study Placebo Group. Arthritis Rheum 2011:63:3078-85.

18 Roth MD, Tseng CH, Clements PJ, et al. Predicting treatment outcomes and responder subsets in scleroderma-related interstitial lung disease. Arthritis Rheum 2011;63:2797-808.

19 Tashkin DP, Elashoff R, Clements PJ, et al. Cyclophosphamide versus placebo in scleroderma lung disease. N Engl J Med 2006:354:2655-66. 